\title{
GAIA Level 3a Failure to Thrive
}

National Cancer Institute

\section{Source}

National Cancer Institute. GAIA Level 3a Failure to Thrive. NCI Thesaurus. Code C128773.

GAIA Level 3a Failure to Thrive is defined by four criteria: first, infants with an undocumented birth date, where age is determined based on mother's recall to the nearest month; second, weight obtained using either beam balance or spring balance scale; third, a minimum of two weights measured at least four weeks apart; fourth, weight for age deceleration must pass through at least two centile spaces on a growth chart. 